\title{
Two characterizations of the shape of the base poset derived from the Lehmer code of a permutation using permutation patterns
}

\author{
Masaya TOMie
}

\begin{abstract}
The Lehmer code is a classical and fundamental permutation code which encodes information about the inversions of a permutation. Denoncourt constructed a poset $M_{\omega}$ which is the subposet of joinirreducible elements of the Lehmer codes of the permutations in $\left[e_{n}, \omega\right]$ in the left weak Bruhat order, i.e., the inversion order, on $S_{n}$ for $\omega \in S_{n}$. In this paper, we investigate the poset structure of $M_{\omega}$ in terms of pattern avoidance. First we show that $M_{\omega}$ is a $B_{2}$-free poset if and only if $\omega$ is a 3412-3421-avoiding permutation. Next we prove that $M_{\omega}$ is poset isomorphic to the corresponding root poset if and only if $\omega$ is a 321-avoiding permutation.
\end{abstract}

AMS 2000 SUBJECT ClASSIFICATIONS: 06A07, 05A05.

KEYwORDS AND PHRASES: Permutation patterns, Lehmer codes, root posets.

\section{Introduction}

The Lehmer code [6] is a classical and fundamental permutation code which encodes information about the inversions of a permutation. The Lehmer code of a permutation $\omega \in S_{n}$ is an $n$-tuple of nonnegative integers. Therefore a set of Lehmer codes has a poset structure under the product order on $\mathbb{N}^{n}$. In [3], Denoncourt investigated the poset structure of the Lehmer codes of permutations in an interval in the left weak Bruhat order, i.e., the inversion order, and he showed that the poset is a distributive lattice. He also described the subposet of the join-irreducible elements of the distributive lattice and denoted it by $M_{\omega}$ for $\omega \in S_{n}$. In this paper, we extend Denoncourt's work. More precisely, we investigate the poset structure of $M_{\omega}$ in terms of pattern avoidance.

In Section 2 we prove that $M_{\omega}$ does not have a subposet isomorphic to Boolean algebra of rank 2 if and only if $\omega$ is a 3412-3421-avoiding permutation. We remark that the number of 3412-3421-avoiding permutations in $S_{n}$ is enumerated by Schröder numbers.

arXiv: 1111.3094 
In Section 3 we discuss a relation between $M_{\omega}$ and the corresponding root poset $\Delta(\omega)$ which we introduce at the beginning of Section 3. The posets $M_{\omega}$ and $\Delta(\omega)$ are both described by using inversions and the number of elements of $M_{\omega}$ and that of $\Delta(\omega)$ are the number of inversions of $\omega$. Hence it is natural to ask how these two structures are related. Indeed we show that $M_{\omega}$ and $\Delta(\omega)$ are order isomorphic if and only if $\omega$ is a 321-avoiding permutation.

In the remaining part of this section we give the definitions of pattern avoidance for permutations and posets. After that we introduce the Lehmer codes and Denoncourt's previous results about the poset structure of Lehmer codes of permutations in an interval.

\subsection{Pattern avoidance for permutations and pattern avoidance for posets}

In this paper we use one-line notation. We denote a permutation $\omega \in S_{n}$ by the sequence $\omega(1) \omega(2) \cdots \omega(n)$. For $\omega \in S_{n}$ and $\pi \in S_{k}$ with $k<n$, we say that a permutation $\omega$ has a $\pi$-pattern if $\operatorname{st}\left(\omega\left(i_{1}\right) \omega\left(i_{2}\right) \cdots \omega\left(i_{k}\right)\right)=$ $\pi(1) \pi(2) \cdots \pi(k)$ for some $1 \leq i_{1}<i_{2}<\cdots<i_{k} \leq n$, where $s t\left(\omega\left(i_{1}\right) \omega\left(i_{2}\right) \cdots \omega\left(i_{k}\right)\right)$ is a permutation in $S_{k}$ defined by the following process: the smallest value of $\omega\left(i_{1}\right) \omega\left(i_{2}\right) \cdots \omega\left(i_{k}\right)$ is replaced with 1 , the secondsmallest value is replaced with 2 , and so on. We call st $\left(\omega\left(i_{1}\right) \omega\left(i_{2}\right) \cdots \omega\left(i_{k}\right)\right)$ the standardization of $\omega\left(i_{1}\right) \omega\left(i_{2}\right) \cdots \omega\left(i_{k}\right)$. If $\operatorname{st}\left(\omega\left(i_{1}\right) \omega\left(i_{2}\right) \cdots \omega\left(i_{k}\right)\right) \neq$ $\pi(1) \pi(2) \cdots \pi(k)$ for any $1 \leq i_{1}<i_{2}<\cdots<i_{k} \leq n$, we say that $\omega$ is a $\pi$-avoiding permutation.

The most popular results on pattern avoidance are the enumerations of permutations avoiding a pattern of length three, which is enumerated by the Catalan numbers [4], [5]. For further information on pattern avoidance, see Bóna's text [2].

Next we introduce pattern avoidance for posets. Let $P$ and $Q$ be posets. A subposet $R \subset P$ is called a $Q$-pattern subposet if $R \simeq Q$ as a poset. We say that $P$ is $Q$-free if $P$ has no $Q$-pattern subposets. One interesting result about pattern avoidance for posets is that the number of $1+3$-free and $2+2$-free posets is enumerated by the Catalan numbers [7]. In this paper we consider $B_{2}$-free posets, where $B_{2}$ is the Boolean algebra of rank 2 .

\subsection{The posets of Lehmer codes}

Let $\operatorname{Inv}(\omega):=\{(i, j) \mid 1 \leq i<j \leq n, \omega(i)>\omega(j)\}$, the set of inversions for $\omega \in S_{n}$, and let $\Lambda_{\omega}:=\{\sigma \mid \operatorname{Inv}(\sigma) \subset \operatorname{Inv}(\omega)\}$. In other words, $\Lambda_{\omega}$ is the 
interval $\left[e_{n}, \omega\right]$, where $e_{n}$ is the identity permutation, in the left weak Bruhat order, i.e., inversion order, on $S_{n}$ [1].

For $i \in[n]:=\{1,2, \cdots, n\}$, let

$$
c_{i}(\omega):=|\{j \mid 1 \leq i<j \leq n, \omega(i)>\omega(j)\}|,
$$

the number of inversions of $\omega \in S_{n}$ with the first coordinate equal to $i$. Then the sequence

$$
\mathbf{c}(\omega)=\left(c_{1}(\omega), c_{2}(\omega), \cdots, c_{n}(\omega)\right)
$$

is called the Lehmer code for $\omega \in S_{n}$. For example, for $\omega=423615$, the inversions are

$$
(1,2),(1,3),(1,5),(2,5),(3,5),(4,5),(4,6)
$$

and we have

$$
c_{1}(\omega)=3, c_{2}(\omega)=1, c_{3}(\omega)=1, c_{4}(\omega)=2, c_{5}(\omega)=0, c_{6}(\omega)=0
$$

and the corresponding Lehmer code is $(3,1,1,2,0,0)$.

For $\omega \in S_{n}$ and $1 \leq i<j \leq n+1$, let

$$
c_{i, j}(\omega):=|\{k \mid i<k<j, \omega(i)>\omega(k)\}|,
$$

the number of inversions $(i, k) \in \operatorname{Inv}(\omega)$ with $i<k<j[3]$.

For $\omega \in S_{n}$, set

$$
\mathbf{c}\left(\Lambda_{\omega}\right):=\left\{\mathbf{c}(\sigma) \mid \sigma \in \Lambda_{\omega}\right\},
$$

the set of Lehmer codes of permutations in $\left[e_{n}, \omega\right]$ in the inversion order. Then $\mathbf{c}\left(\Lambda_{\omega}\right) \subset \mathbb{N}^{n}$ and $\mathbf{c}\left(\Lambda_{\omega}\right)$ is a subposet of $\mathbb{N}^{n}$ with the obvious product order. Denoncourt proved the following result.

Theorem $1.1([3])$. For $\omega \in S_{n}$, the subposet $\mathbf{c}\left(\Lambda_{\omega}\right)$ of $\mathbb{N}^{n}$ is a distributive lattice.

Let $L$ be a distributive lattice and let $P$ be the subposet of joinirreducibles of $L$. Then $L$ is poset isomorphic to $J(P)$, where $J(P)$ is the poset of order ideals of $P$ ordered by inclusion, see Theorem 3.4.1 in [7]. Denoncourt determined the subposet of join-irreducible elements in $\mathbf{c}\left(\Lambda_{\omega}\right)$ [3]. We denote the $j$-th coordinate of $\boldsymbol{x} \in \mathbb{N}^{n}$ by $\pi_{j}(\boldsymbol{x})$. 
Definition $1.1([3])$. For $i \in[n]$ such that $c_{i}(\omega)>0$ and for each $x \in$ $\left[c_{i}(\omega)\right]$, define $m_{i, x}(\omega) \in \mathbb{N}^{n}$ coordinate-wise by

1. $\pi_{j}\left(m_{i, x}(\omega)\right):=0$, if $j<i$,

2. $\pi_{j}\left(m_{i, x}(\omega)\right):=x$, if $j=i$,

3. $\pi_{j}\left(m_{i, x}(\omega)\right):=0$, if $j>i$ and $(i, j) \in \operatorname{Inv}(\omega)$, i.e., $\omega(j)<\omega(i)$,

4. $\pi_{j}\left(m_{i, x}(\omega)\right):=\max \left\{0, x-c_{i, j}(\omega)\right\}$, if $j>i$ and $(i, j) \notin \operatorname{Inv}(\omega)$, i.e., $\omega(j)>\omega(i)$.

In other words, if $j>i$ and $\omega(j)>\omega(i)$, then the $j$-th coordinate is equal to $x$ minus the number of 0 entries between $i$-th coordinate and $j$-th coordinate, if not negative, or 0 .

For $\omega \in S_{n}$, Denoncourt set

$$
M_{\omega}:=\left\{m_{i, x}(\omega) \mid 1 \leq i \leq n, c_{i}(\omega)>0, x \in\left[c_{i}(\omega)\right]\right\}
$$

which is a subposet of $\mathbb{N}^{n}$ in the product order. Denoncourt provided the following result [3].

Theorem $1.2([3])$. For $\omega \in S_{n}, M_{\omega}$ is the subposet of join-irreducibles for $\mathbf{c}\left(\Lambda_{\omega}\right)$.

If $\omega=5371642$, then we have

$$
\begin{array}{ll}
m_{1,4}(5371642)=(4,0,3,0,2,0,0), & m_{1,3}(5371642)=(3,0,2,0,1,0,0), \\
m_{1,2}(5371642)=(2,0,1,0,0,0,0), & m_{1,1}(5371642)=(1,0,0,0,0,0,0), \\
m_{2,2}(5371642)=(0,2,2,0,1,1,0), & m_{2,1}(5371642)=(0,1,1,0,0,0,0), \\
m_{3,4}(5371642)=(0,0,4,0,0,0,0), & m_{3,3}(5371642)=(0,0,3,0,0,0,0), \\
m_{3,2}(5371642)=(0,0,2,0,0,0,0), & m_{3,1}(5371642)=(0,0,1,0,0,0,0), \\
m_{5,2}(5371642)=(0,0,0,0,2,0,0), & m_{5,1}(5371642)=(0,0,0,0,1,0,0), \\
m_{6,1}(5371642)=(0,0,0,0,0,1,0), &
\end{array}
$$

and $\mathbf{c}(\omega)=(\mathbf{4}, \mathbf{2}, \mathbf{4}, \mathbf{0}, \mathbf{2}, \mathbf{1}, \mathbf{0})$. Figure 1 shows the Hasse diagram of $M_{5371642}$.

Denoncourt set

$$
C_{i}(\omega):=\left\{m_{i, x}(\omega) \mid x \in\left[c_{i}(\omega)\right]\right\},
$$

for $1 \leq i \leq n$ with $c_{i}(\omega) \neq 0$. The following results of Denoncourt will be required for the proofs of our results. 


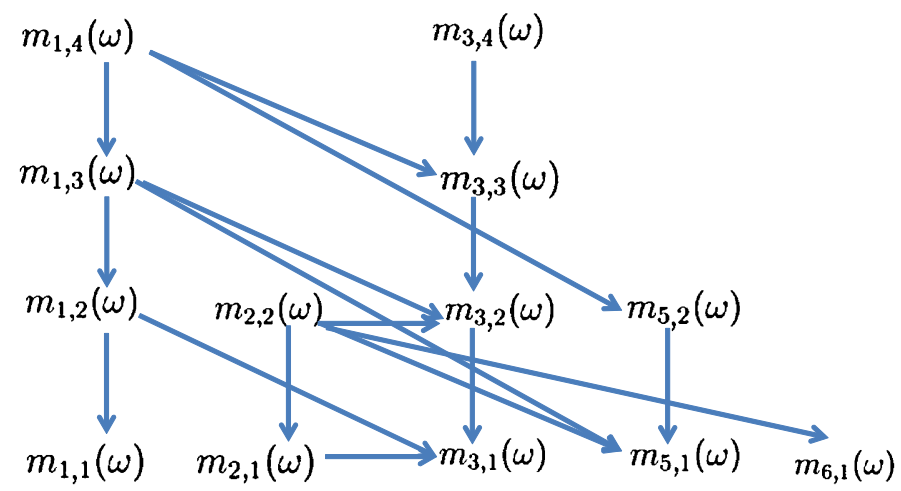

Figure 1: Hasse diagram of $M_{\omega}$ with $\omega=5371642$.

Lemma $1.1([3])$. Let $\omega \in S_{n}$. For $1 \leq i<j \leq n$ with $x \in\left[c_{i}(\omega)\right]$ and $y \in\left[c_{j}(\omega)\right]$, we have $m_{i, x}(\omega) \not \leq m_{j, y}(\omega)$. If $m_{i, x}(\omega)>m_{j, y}(\omega)$ for some $1 \leq i \neq j \leq n, x \in\left[c_{i}(\omega)\right]$ and $y \in\left[c_{j}(\omega)\right]$, then $i<j$. ([3] Lemma 4.11.)

Lemma $1.2([3])$. Let $\omega \in S_{n}$. For $1 \leq i \leq n$ with $c_{i}(\omega) \neq 0$ and $1 \leq$ $y \leq x \leq c_{i}(\omega)$, we have $m_{i, y}(\omega) \leq m_{i, x}(\omega)$ and hence $C_{i}(\omega)$ is a chain. ([3] Lemma 4.10.)

Lemma $1.3([3])$. Let $\omega \in S_{n}$. For $1 \leq i<j \leq n$ with $(i, j) \in \operatorname{Inv}(\omega)$, every element of $C_{i}(\omega)$ is incomparable with every element of $C_{j}(\omega)$. Equivalently, if $m_{i, x}(\omega)>m_{i, y}(\omega)$ for some $m_{i, x}(\omega) \in C_{i}(\omega)$ and $m_{j, y}(\omega) \in C_{j}(\omega)$ with $1 \leq i<j \leq n$, then $\omega(i)<\omega(j)$. ([3] Lemma 4.12.)

Lemma $1.4([3])$. For $\omega \in S_{n}$ and $1 \leq i<j \leq n$ with $\omega(i)<\omega(j)$, we have $c_{i}(\omega) \leq c_{j}(\omega)+c_{i, j}(\omega)$. ([3] Lemma 2.6.)

The following statement is a trivial consequence of Definition 1.1, hence we omit the proof.

Lemma 1.5. Let $\omega \in S_{n}$. For $1 \leq i<j \leq n$ with $\omega(i)<\omega(j)$, suppose that the $j$-th coordinate of $m_{i, x}(\omega)$ is $z \geq 1$. Then $c_{i, j}(\omega)$, the number of $i<k<j$ such that $\omega(i)>\omega(k)$, is equal to the number of 0 between $i$-th coordinate and $j$-th coordinate, and hence equals $x-z$.

We can write

$$
m_{i, x}(\omega)=\left(0, \cdots, 0, \stackrel{i-\text { th }}{x}, \cdots,{ }^{j-\text { th }}, \cdots, 0\right)
$$

for some $z \geq 1$. 
Proposition 1.1. If $\omega$ is a 231-avoiding permutation then $M_{\omega}$ is a poset which is the disjoint union of chains.

Proof. For $m_{i, x}(\omega), m_{j, y}(\omega) \in M_{\omega}$ with $1 \leq i<j \leq n, x \in\left[c_{i}(\omega)\right]$ and $y \in\left[c_{j}(\omega)\right]$, if $(i, j) \in \operatorname{Inv}(\omega)$, then $m_{i, x}(\omega)$ and $m_{j, y}(\omega)$ are incomparable by Lemma 1.3. Suppose that $(i, j) \notin \operatorname{Inv}(\omega)$, then $\omega(i)<\omega(k)$ for all $j<k \leq n$, because $\omega$ is a 231-avoiding permutation. The $j$-th coordinate of $m_{i, x}(\omega)$ is 0 and hence $m_{i, x}(\omega)$ and $m_{j, y}(\omega)$ are incomparable. Now $m_{i, x}(\omega)$ and $m_{j, y}(\omega)$ are incomparable for $1 \leq i<j \leq n$ and therefore $M_{\omega}$ is a disjoint union of chains.

The converse is false. For example, if $\omega=4231$, then $\omega$ has a 231-pattern and $M_{4231}$ is disjoint union of 2 chains.

Remark 1.1. When we write $m_{i, x}(\omega)$ for $1 \leq i \leq n$ and $x \in\left[c_{i}(\omega)\right]$, we always assume that $c_{i}(\omega)>0$.

\section{A relation between the shape of $M_{\omega}$ and the corresponding permutation $\omega$}

In this section we discuss the shape of $M_{\omega}$ in terms of pattern avoidance.

Definition 2.1. Let $P$ be a poset. $A$ 4-element subposet $\{a, b, c, d\} \subset P$ is called a $B_{2}$-pattern subposet if $\{a, b, c, d\} \simeq B_{2}$, where $B_{2}$ is a Boolean algebra of rank 2. We say that $P$ is a $B_{2}$-free poset if there are no 4-element subposets isomorphic to $B_{2}$.

Next we define a parallelogram pattern subposet and a pseudo parallelogram pattern subposet.

Definition 2.2. Suppose $1 \leq i<j \leq n, a<b \in\left[c_{i}(\omega)\right]$ and $c<d \in\left[c_{j}(\omega)\right]$ with $a+d=b+c$.

1. A subposet $\left\{m_{i, a}(\omega), m_{i, b}(\omega), m_{j, c}(\omega), m_{j, d}(\omega)\right\}$ is called a parallelogram pattern subposet if

$$
m_{i, a}(\omega)>m_{j, c}(\omega) \text { and } m_{i, b}(\omega)>m_{j, d}(\omega)
$$

in $M_{\omega}$ and $m_{i, a}(\omega)$ and $m_{j, d}(\omega)$ are incomparable. We say that $M_{\omega}$ has a parallelogram pattern if $M_{\omega}$ contains a parallelogram pattern subposet.

2. A subposet $\left\{m_{i, a}(\omega), m_{i, b}(\omega), m_{j, c}(\omega), m_{j, d}(\omega)\right\}$ is called a pseudo parallelogram pattern subposet if

$$
m_{i, a}(\omega)>m_{j, c}(\omega) \text { and } m_{i, b}(\omega)>m_{j, d}(\omega)
$$




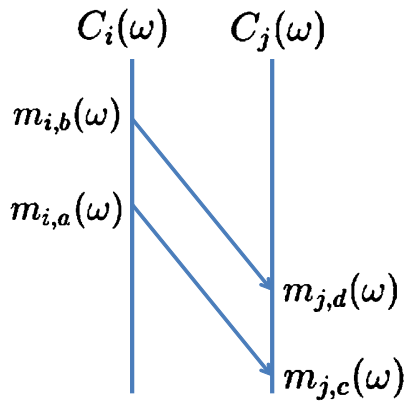

Parallelogram Pattern

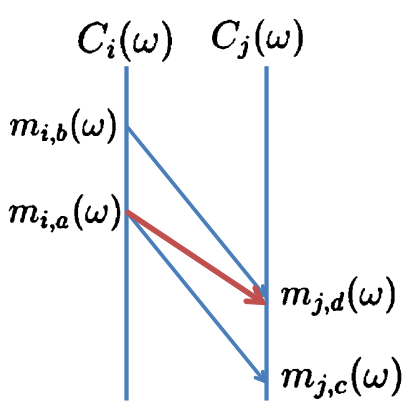

Pseudo Parallelogram Pattern

Figure 2: Illustration of a parallelogram pattern and a pseudo parallelogram pattern.

in $M_{\omega}$ and $m_{i, a}(\omega)$ and $m_{j, d}(\omega)$ are comparable. We say that $M_{\omega}$ has a pseudo parallelogram pattern if $M_{\omega}$ contains a pseudo parallelogram pattern subposet.

The left-hand side of Figure 2 shows a visualization of a parallelogram pattern and the right-hand side shows a visualization of a pseudo parallelogram pattern.

Remark 2.1. A parallelogram pattern subposet is isomorphic to $B_{2}$, the Boolean algebra of rank 2 .

Remark 2.2. Notation is as above, if $\left\{m_{i, a}(\omega), m_{i, b}(\omega), m_{j, c}(\omega), m_{j, d}(\omega)\right\}$ is a pseudo parallelogram pattern subposet, then we have

$$
m_{i, b}(\omega)>m_{i, a}(\omega)>m_{j, d}(\omega)>m_{j, c}(\omega)
$$

in $M_{\omega}$ by Lemma 1.1.

The following Lemma is a straightforward consequence of Definition 1.1.

Lemma 2.1. For $\omega \in S_{n}, 1 \leq i \leq n$ and $x \in\left[c_{i}(\omega)\right]$, we have

1. $\pi_{j}\left(m_{i, x}(\omega)\right)=0$, if $j<i$,

2. $\pi_{i}\left(m_{i, x}(\omega)\right)=x$,

3. $\pi_{j}\left(m_{i, x}(\omega)\right)=0$, if $j>i$ and $j \in\left\{i_{1}, i_{2}, \cdots, i_{x}\right\}$,

4. $\pi_{j}\left(m_{i, x}(\omega)\right)=x-\left|\left\{k \mid i_{k}<j, 1 \leq k \leq x\right\}\right|$, if $j>i$ and $j \notin$ $\left\{i_{1}, i_{2}, \cdots, i_{x}\right\}$, 

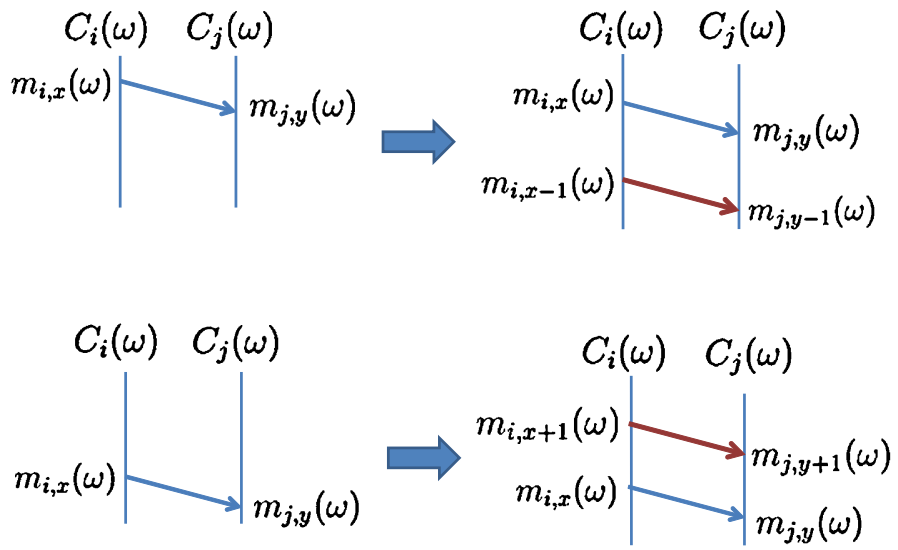

Figure 3: A visualization of Lemma 2.2.

and we can write

$$
\begin{array}{r}
m_{i, x}(\omega)=\left(0, \cdots, 0, \stackrel{i-\text { th }}{x}, x, \cdots, x, \stackrel{i_{1}-\text { th }}{0}, x-1, \cdots, x-1, \stackrel{i_{2}-\text { th }}{0}, x-2, \cdots,\right. \\
\left.x-2, \cdots, 3, \stackrel{i_{x-2}-\text { th }}{0}, 2, \cdots, 2, \stackrel{i_{x-1}-\text { th }}{0}, 1, \cdots, 1, \stackrel{i_{x}-\text { th }}{0}, 0, \cdots, 0\right),
\end{array}
$$

where $\left(i, i_{1}\right),\left(i, i_{2}\right), \cdots,\left(i, i_{x}\right)$ are the inversions of $\omega$ involving $i$ with $i<$ $i_{1}<\cdots<i_{x} \leq n$.

The following fact easily follows from Lemma 2.1.

Lemma 2.2. Let $\omega \in S_{n}, 1 \leq i<j \leq n, x \in\left[c_{i}(\omega)\right]$ and $y \in\left[c_{j}(\omega)\right]$.

1. If $x, y \geq 2$ and $m_{i, x}(\omega)>m_{j, y}(\omega)$ in $M_{\omega}$, then $m_{i,(x-1)}(\omega)>$ $m_{j,(y-1)}(\omega)$.

2. If $x<c_{i}(\omega), y<c_{j}(\omega)$ and $m_{i, x}(\omega)>m_{j, y}(\omega)$, then $m_{i,(x+1)}(\omega)>$ $m_{j,(y+1)}(\omega)$.

Lemma 2.3. For $\omega \in S_{n}, 1 \leq i<j \leq n, x \in\left[c_{i}(\omega)\right]$ and $y \in\left[c_{j}(\omega)\right]$, if $m_{i, x}(\omega)>m_{j, y}(\omega)$ in $M_{\omega}$, then we have $c_{j}(\omega)+x \geq c_{i}(\omega)+y$ and $x \geq y$.

Figure 4 shows a visualization of Lemma 2.3.

Proof. We can write

$$
m_{i, x}(\omega)=(0, \cdots, 0, \stackrel{i-\text { th }}{x}, \cdots, \stackrel{j-\text { th }}{z}, \cdots, 0)
$$




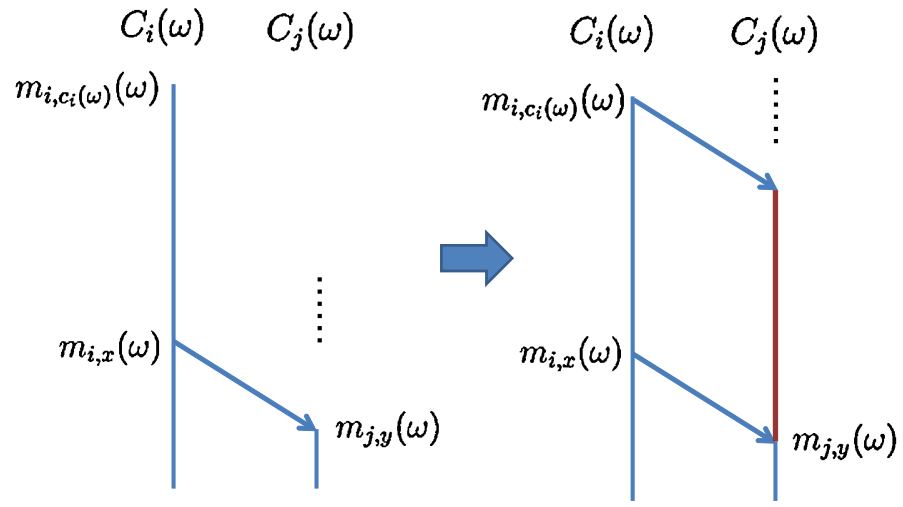

Figure 4: A visualization of Lemma 2.3.

and

$$
m_{j, y}(\omega)=(0, \cdots, 0, \stackrel{i-\text { th }}{0}, \cdots, 0, \stackrel{j-\text { th }}{y}, \cdots, 0)
$$

for some $z \geq y \geq 1$. Obviously, we have $x \geq z \geq y$. By Lemma 1.3, we see $\omega(i)<\omega(j)$. Also $c_{i, j}(\omega)=x-z \leq x-y$ by Lemma 1.5. From Lemma 1.4, we obtain $c_{j}(\omega) \geq c_{i}(\omega)-c_{i, j}(\omega)$ and hence $c_{j}(\omega) \geq c_{i}(\omega)-(x-y)$. We have $c_{j}(\omega)+x \geq c_{i}(\omega)+y$.

Remark 2.3. The above Lemma 2.2 and Lemma 2.3 show that if $m_{i, x}(\omega)>$ $m_{j, y}(\omega)$, and notation is as above, then $x \geq y, c_{i}(\omega)+y-x \in\left[c_{j}(\omega)\right]$,

$$
\begin{aligned}
m_{i, x+1}(\omega)>m_{j, y+1}(\omega), & m_{i, x+2}(\omega)>m_{j, y+2}(\omega), \cdots, \\
& m_{i, c_{i}(\omega)}(\omega)>m_{j, c_{i}(\omega)+y-x}(\omega)
\end{aligned}
$$

and

$$
\begin{array}{r}
m_{i, x-1}(\omega)>m_{j, y-1}(\omega), m_{i, x-2}(\omega)>m_{j, y-2}(\omega), \cdots, \\
m_{i, 1+x-y}(\omega)>m_{j, 1}(\omega) .
\end{array}
$$

Figure 5 shows a visualization of Remark 2.3.

Proposition 2.1. For $\omega \in S_{n}$, if $M_{\omega}$ has a pseudo parallelogram pattern, then $M_{\omega}$ has a parallelogram pattern.

Proof. Let $\left\{m_{i, a}(\omega), m_{i, b}(\omega), m_{j, c}(\omega), m_{j, d}(\omega)\right\} \subset M_{\omega}$ be a pseudo parallelogram pattern subposet such that $m_{i, b}(\omega)>m_{i, a}(\omega)>m_{j, d}(\omega)>m_{j, c}(\omega)$ with $1 \leq i<j \leq n, a<b \in\left[c_{i}(\omega)\right]$ and $c<d \in\left[c_{i}(\omega)\right]$ with $a+d=b+c$. One will find that $a \geq d$. 


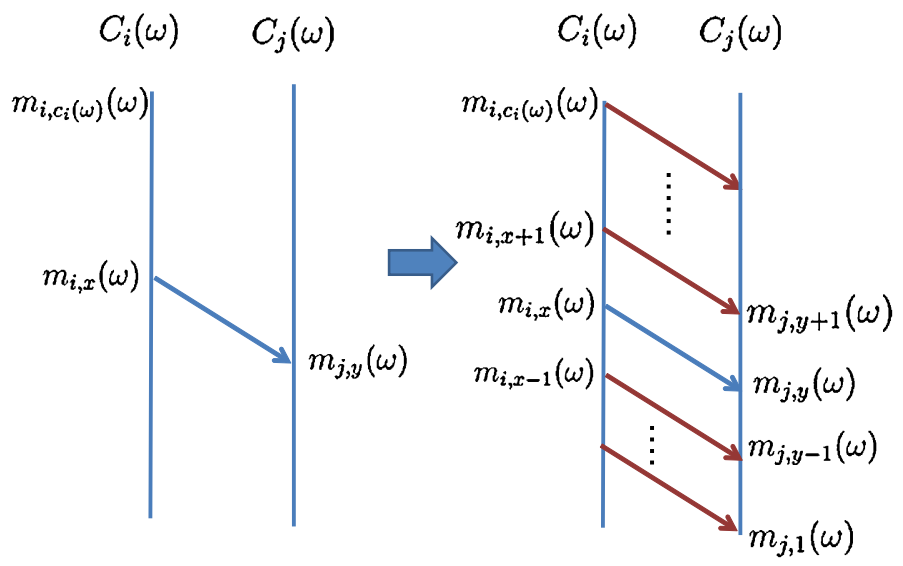

Figure 5: A visualization of Remark 2.3.

We will prove the existence of a parallelogram pattern subposet by induction on $k=a-d$.

Suppose that $a=d$. We show that $\left\{m_{i, b}(\omega), m_{i, a}(\omega), m_{j, a+1}(\omega)\right.$, $\left.m_{j, c+1}(\omega)\right\}$ is a parallelogram pattern subposet. Because $m_{i, a}(\omega)>m_{j, a}(\omega)$ and $a<b \in\left[c_{i}(\omega)\right]$, we have $m_{j, a+1}(\omega) \in M_{\omega}$ and $m_{i, b}(\omega) \geq m_{i, a+1}(\omega)>$ $m_{j, a+1}(\omega)$ by Remark 2.3. Also we can see $m_{i, a}(\omega)>m_{j, a}(\omega)=m_{j, d}(\omega) \geq$ $m_{j, c+1}(\omega)$ and hence $m_{i, a}(\omega)>m_{j, c+1}(\omega)$. We find that $m_{i, a}(\omega)$ and $m_{j, a+1}(\omega)$ are incomparable by Lemma 2.3. Therefore $\left\{m_{i, b}(\omega), m_{i, a}(\omega)\right.$, $\left.m_{j, a+1}(\omega), m_{j, c}(\omega)\right\}$ is a parallelogram pattern subposet.

Let $a-d=k \geq 1$. We consider a subposet $\left\{m_{i, b}(\omega), m_{i, a}(\omega)\right.$, $\left.m_{j, d+1}(\omega), m_{j, c+1}(\omega)\right\} \subset M_{\omega}$, where $i, j, a, b, c$ and $d$ are as above. Because $m_{i, a}(\omega)>m_{j, d}(\omega), a<b \in\left[c_{i}(\omega)\right]$ and $m_{j, d+1}(\omega) \in M_{\omega}$, we have $m_{i, b}(\omega) \geq$ $m_{i, a+1}(\omega)>m_{j, d+1}(\omega)$ by Remark 2.3. Also we can see $m_{i, a}(\omega)>m_{j, d}(\omega) \geq$ $m_{j, c+1}(\omega)$.

If $m_{i, a}(\omega)>m_{j, d+1}(\omega)$, then there exists a parallelogram pattern subposet of $M_{\omega}$ by the induction hypothesis.

If $m_{i, a}(\omega)$ and $m_{j, d+1}(\omega)$ are incomparable in $M_{\omega}$, then $\left\{m_{i, b}(\omega), m_{i, a}(\omega)\right.$, $\left.m_{j, d+1}(\omega), m_{j, c+1}(\omega)\right\} \subset M_{\omega}$ is a parallelogram pattern subposet.

Now we conclude that if $M_{\omega}$ has a pseudo parallelogram pattern, then $M_{\omega}$ contains a parallelogram pattern.

Figure 6 shows a visualization of the argument.

Proposition 2.2. For $\omega \in S_{n}$, the poset $M_{\omega}$ has a $B_{2}$-pattern subposet if and only if $M_{\omega}$ has a parallelogram pattern subposet. 


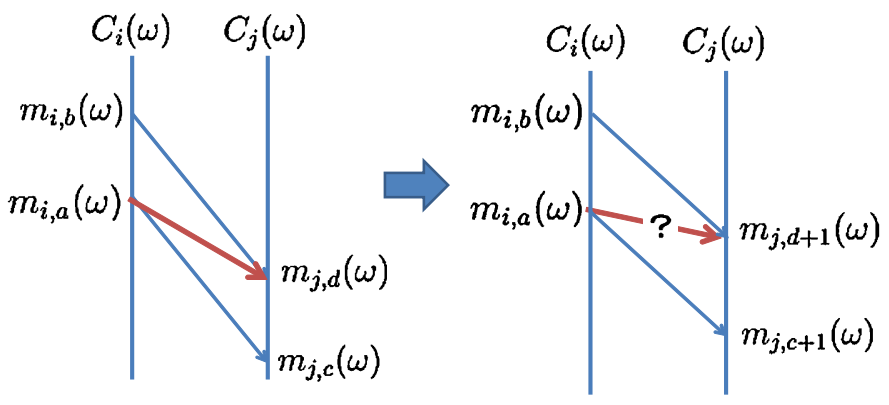

Figure 6: Illustration of the proof of Proposition 2.1.

Proof. If $M_{\omega}$ has a parallelogram pattern subposet, then obviously $M_{\omega}$ has a $B_{2}$-pattern subposet.

Conversely suppose that $M_{\omega}$ has a $B_{2}$-pattern subposet. Let $\left\{m_{i, a}(\omega)\right.$, $\left.m_{j, b}(\omega), m_{k, c}(\omega), m_{l, d}(\omega)\right\}$ with $i, j, k, l \in \mathbb{N}, a \in\left[c_{i}(\omega)\right], b \in\left[c_{j}(\omega)\right], c \in$ $\left[c_{k}(\omega)\right]$ and $d \in\left[c_{l}(\omega)\right]$ be a $B_{2}$-pattern subposet of $M_{\omega}$. Without loss of generality, we can assume that $m_{i, a}(\omega)$ (resp. $m_{l, d}(\omega)$ ) is the maximum (resp. minimum) element and $m_{j, b}(\omega)$ and $m_{k, c}(\omega)$ are incomparable with $j<k$ and hence we can set $\left\{m_{i, a}(\omega), m_{j, b}(\omega), m_{k, c}(\omega), m_{l, d}(\omega)\right\}$ with $1 \leq i \leq j<$ $k \leq l \leq n$.

It suffices to prove the existence of a pseudo parallelogram pattern subposet or a parallelogram pattern subposet by Proposition 2.1. We give a careful case-by-case proof.

Case 1: $d \geq 2$.

Because $m_{i, a}(\omega)>m_{l, d}(\omega)$ and Remark 2.3, we have $a \geq d \geq 2$ and $m_{i, a-1}(\omega)>m_{l, d-1}(\omega)$ in $M_{\omega}$. Then the subposet $\left\{m_{i, a}(\omega), m_{i, a-1}(\omega)\right.$, $\left.m_{l, d}(\omega), m_{l, d-1}(\omega)\right\} \subset M_{\omega}$ is either a parallelogram pattern subposet or a pseudo parallelogram pattern subposet.

Case 2: $d=1$ and $i=j$.

Because $m_{i, a}(\omega)>m_{i, b}(\omega)>m_{l, 1}(\omega)=m_{l, d}(\omega)$ and Remark 2.3, we have $a \geq b+1$ and $m_{i, b+1}(\omega)>m_{l, 2}(\omega)$ in $M_{\omega}$. Then the subposet $\left\{m_{i, b+1}(\omega)\right.$, $\left.m_{i, b}(\omega), m_{l, 2}(\omega), m_{l, 1}(\omega)\right\} \subset M_{\omega}$ is either a parallelogram pattern subposet or a pseudo parallelogram pattern subposet.

Case 3: $d=1$ and $k=l$.

Because $m_{i, a}(\omega)>m_{k, c}(\omega)>m_{l, 1}(\omega)=m_{k, 1}(\omega)$ and by Lemma 2.3 and Remark 2.3, we have $a \geq c \geq 2$ and $m_{i, a-1}(\omega)>m_{k, c-1}(\omega)$ in $M_{\omega}$. Then the subposet $\left\{m_{i, a}(\omega), m_{i, a-1}(\omega), m_{k, c}(\omega), m_{k, c-1}(\omega)\right\}$ is either a parallelogram pattern subposet or a pseudo parallelogram pattern subposet. 


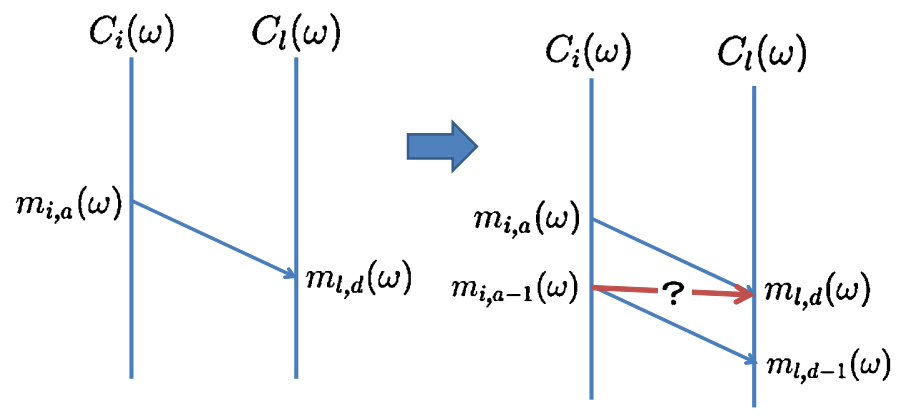

Figure 7: Illustration of Case 1.

The remaining cases are the case of $d=1$ with $i<j<k<l$.

Case 4: $b \geq 2$ or $c \geq 2$.

Suppose $b \geq 2$, we have $a \geq 2$ by Lemma 2.3. Also we see $m_{i, a-1}(\omega)>$ $m_{j, b-1}(\omega)$ because $m_{i, a}(\omega)>m_{j, b}(\omega)$ with $a, b \geq 2$ and Lemma 2.2. Then the subposet $\left\{m_{i, a}(\omega), m_{i, a-1}(\omega), m_{j, b}(\omega), m_{j, b-1}(\omega)\right\}$ is either a parallelogram pattern subposet or a pseudo parallelogram pattern subposet. We can use the same argument for the case of $c \geq 2$.

Case 5: $b=c=1$ and $c_{j}(\omega) \geq 2$ or $c_{k}(\omega) \geq 2$.

Suppose $c_{j}(\omega) \geq 2$. We have $c_{l}(\omega) \geq 2$ and $m_{j, 2}(\omega)>m_{l, 2}(\omega)$ because $m_{j, 1}(\omega)>m_{l, 1}(\omega)$ and by Lemma 2.3 and Remark 2.3. Then the subposet $\left\{m_{j, 2}(\omega), m_{j, 1}(\omega), m_{l, 2}(\omega), m_{l, 1}(\omega)\right\}$ is either a parallelogram pattern subposet or a pseudo parallelogram pattern subposet. We can use the same argument for the case of $c_{k}(\omega) \geq 2$.

Case 6: $b=c=1$ and $c_{j}(\omega)=c_{k}(\omega)=1$.

Because $m_{i, a}(\omega)>m_{j, 1}(\omega)>m_{l, 1}(\omega)$ (resp. $m_{i, a}(\omega)>m_{k, 1}(\omega)>$ $\left.m_{l, 1}(\omega)\right)$ and Lemma 1.3, we have $\omega(i)<\omega(j)<\omega(l)$ (resp. $\omega(i)<\omega(k)<$ $\omega(l)$ ), see Figure 8. We see $\omega(j)<\omega(k)$ because if $\omega(j)>\omega(k)$, then $c_{j}(\omega)$ is larger than $c_{k}(\omega)$, which contradicts the assumption that $c_{j}(\omega)=c_{k}(\omega)=1$. Hence we obtain $\omega(i)<\omega(j)<\omega(k)<\omega(l)$. There exists a unique $p$ such that $j<p \leq n$ and $\omega(j)>\omega(p)$ because $c_{j}(\omega)=1$.

If $j<p<l$, then we see

$$
\begin{array}{r}
m_{j, 1}(\omega)=(0, \cdots, 0, \stackrel{j-\text { th }}{1}, 1, \cdots, 1, \stackrel{p-\text { th }}{0}, \cdots, 0, \stackrel{l-\text { th }}{0}, 0, \cdots, 0), \\
m_{l, 1}(\omega)=(0, \cdots, 0, \stackrel{j-\text { th }}{0}, 0, \cdots, 0, \stackrel{p-\text { th }}{0}, \cdots, 0, \stackrel{l-\text { th }}{1}, 1, \cdots 1,0, \cdots, 0)
\end{array}
$$




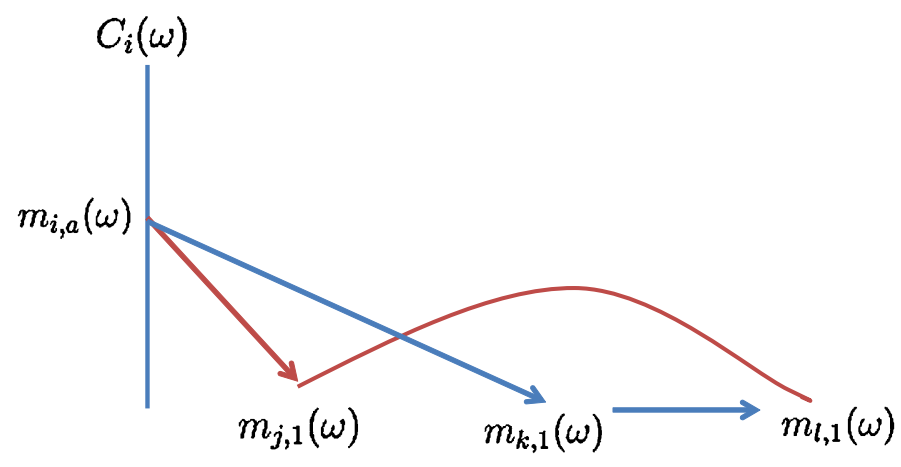

Figure 8: Illustration of Case 6.

and $m_{j, 1}(\omega)$ and $m_{l, 1}(\omega)$ are incomparable. This contradicts the assumption and hence $l<p \leq n$.

For $l<p \leq n,(j, p)$ (resp. $(k, p))$ is the unique inversion of $\omega$ whose first coordinate equals $j$ (resp. $k$ ). Then we can write

$m_{j, 1}(\omega)=(0, \cdots, 0, \stackrel{j-\text { th }}{1}, 1, \cdots, 1, \stackrel{k-\text { th }}{1}, 1, \cdots, 1, \stackrel{l-\text { th }}{1}, 1, \cdots 1, \stackrel{p-\text { th }}{0}, 0, \cdots, 0)$,

$$
m_{k, 1}(\omega)=(0, \cdots, 0, \stackrel{j-\text { th }}{0}, 0, \cdots, 0, \stackrel{k-\text { th }}{1}, 1, \cdots, 1, \stackrel{l-\text { th }}{1}, 1, \cdots 1, \stackrel{p-\text { th }}{0}, 0, \cdots, 0)
$$

and hence $m_{j, 1}(\omega)>m_{k, 1}(\omega)$. Therefore $m_{j, 1}(\omega)$ and $m_{k, 1}(\omega)$ are comparable. This contradicts the assumption. Therefore Case 6 never happens.

From the above argument, we can conclude that if $M_{\omega}$ has a $B_{2}$ pattern, then $M_{\omega}$ contains a pseudo parallelogram pattern or a parallelogram pattern and therefore $M_{\omega}$ has a parallelogram pattern by Proposition 2.1.

Proposition 2.3. For $\omega \in S_{n}$, the poset $M_{\omega}$ has a parallelogram pattern subposet if and only if $\omega$ has a 3412-pattern or a 3421-pattern.

Proof. Suppose that $\omega$ has a 3412-pattern. We will use the same argument if $\omega$ has a 3421-pattern. There exists $1 \leq i<j<k<l \leq n$ such that $s t(\omega(i) \omega(j) \omega(k) \omega(l))=3412$ and we have $c_{i}(\omega) \geq 2$ and $c_{j}(\omega) \geq 2$. We construct a parallelogram pattern subposet or a pseudo parallelogram pattern subposet. Now $(i, k),(i, l),(j, k)$ and $(j, l)$ are inversions of $\omega$ and the $j$-th coordinate of $m_{i, c_{i}(\omega)}(\omega)$ is greater than or equal to 2 by Lemma 2.1. 
We can write

$$
m_{i, c_{i}(\omega)}(\omega)=\left(0, \cdots, 0, c_{i}(\omega), \cdots, \stackrel{j-\text { th }}{x}, \cdots, \stackrel{k-\text { th }}{0}, \cdots, \quad, \quad 0, \cdots 0\right),
$$

$$
\begin{array}{r}
m_{i, c_{i}(\omega)-1}(\omega)=\left(0, \cdots, 0, c_{i}(\omega)-1, \cdots, x-1, \cdots, \quad{ }^{k-\text { th }}, \cdots, 0,0, \cdots 0\right), \\
24) \quad m_{j, 2}(\omega)=(0, \cdots, 0,0, \cdots, 2, \cdots, 0, \cdots, 0, \cdots 0)
\end{array}
$$

and

$$
m_{j, 1}(\omega)=\left(0, \cdots, 0,0,0, \cdots,{ }^{i-\text { th }}, 1, \cdots, 0,0, \cdots, 0,0, \cdots 0\right)
$$

for some $x \geq 2$.

Next we will show that $m_{i, c_{i}(\omega)-1}(\omega)>m_{j, 1}(\omega)$ in $M_{\omega}$.

Let $p$ be the minimum number such that $(j, p)$ is an inversion of $\omega$, such a number exists because $c_{j}(\omega) \geq 2$ and also we have $p \leq k$.

By Lemma 2.1, we can write

$$
\begin{array}{r}
m_{j, 1}(\omega)=\left(0, \cdots, 0, \stackrel{i-\text { th }}{0}, \cdots, 0, \begin{array}{c}
j-\text { th } \\
1
\end{array}, 1 \cdots, 1, \stackrel{p-\text { th }}{0}, 0, \cdots\right. \\
0, \quad 0, \cdots, 0,0, \cdots 0) .
\end{array}
$$

For $q<j$ or $p \leq q$, the $q$-th coordinate of $m_{j, 1}(\omega)$, which is equal to 0 , is less than or equal to that of $m_{i, c_{i}(\omega)-1}(\omega)$. For $j \leq q<p$, the $q$-th coordinate of $m_{j, 1}(\omega)$ equals 1 . Also we see that the $q$-th coordinate of $m_{i, c_{i}(\omega)-1}(\omega)$ equals $x-1$, which is greater than or equal to 1 , because $\omega(i)<\omega(j)<\omega(j+1)<\cdots<\omega(q)<\cdots<\omega(p-1)$ and by Lemma 2.1. Hence we obtain $m_{i, c_{i}(\omega)-1}(\omega)>m_{j, 1}(\omega)$ in $M_{\omega}$.

For a subposet $\left\{m_{i, c_{i}(\omega)}(\omega), m_{i, c_{i}(\omega)-1}(\omega), m_{j, 2}(\omega), m_{j, 1}(\omega)\right\}$, we have $m_{i, c_{i}(\omega)-1}(\omega)>m_{j, 1}(\omega)$ and $m_{i, c_{i}(\omega)}(\omega)>m_{j, 2}(\omega)$ by Lemma 2.2. Hence this subposet is a parallelogram pattern subposet or a pseudo parallelogram pattern subposet. Therefore $M_{\omega}$ has a parallelogram pattern subposet by Proposition 2.1.

Also if $\omega$ has a 3421-pattern, then $M_{\omega}$ has a parallelogram pattern by the same argument.

Conversely suppose that $M_{\omega}$ has a parallelogram pattern subposet $\left\{m_{i, a}(\omega), m_{i, b}(\omega), m_{j, c}(\omega), m_{j, d}(\omega)\right\}$ with $1 \leq i<j \leq n, a<b \in\left[c_{i}(\omega)\right]$, $c<d \in\left[c_{j}(\omega)\right]$ and $a+d=b+c$, where $m_{i, b}(\omega)$ (resp. $\left.m_{j, c}(\omega)\right)$ is the maximum element (resp. minimum element) and $m_{i, a}(\omega)$ and $m_{j, d}(\omega)$ are 
incomparable. We remark that $d \geq 2$ because $d>c \geq 1$. Also we find $\omega(i)<\omega(j)$ by Lemma 1.3 .

We can write

$$
m_{i, b}(\omega)=(0, \cdots, 0, \stackrel{i-\text { th }}{b}, \cdots, \stackrel{j-\text { th }}{z}, \cdots \cdots, 0)
$$

and

$$
m_{j, d}(\omega)=(0, \cdots, 0, \stackrel{i-\text { th }}{0}, \cdots, \quad d-\text { th }, \cdots, 0)
$$

for some $z \geq d \geq 2$.

The $j$-th coordinate of $m_{i, b}(\omega)$, which is equal to $z$, is greater than or equal to 2. By Lemma 2.1, there exists $j<k<l \leq n$ such that $(i, k)$ and $(i, l)$ are inversions of $\omega$. Therefore we obtain $s t(\omega(i) \omega(j) \omega(k) \omega(l))=3412$ or 3421 .

Now we conclude that if $M_{\omega}$ contains a parallelogram pattern, then $\omega$ has a 3412-pattern or a 3421-pattern.

From Proposition 2.2 and Proposition 2.3, we obtain the following result.

Theorem 2.1. For $\omega \in S_{n}$, the poset $M_{\omega}$ is a $B_{2}$-free poset if and only if $\omega$ is a 3412-3421-avoiding permutation.

\section{A relation between $M_{\omega}$ and the corresponding root poset}

In this section we endow the set of inversions of a permutation $\omega$ with a partial order $\preceq$ such that

$$
(p, q) \preceq(r, s) \Longleftrightarrow[p, q] \subset[r, s]
$$

where $1 \leq p, q, r, s \leq n$ and $(p, q)$ and $(r, s)$ are inversions of $\omega$. Note that $[p, q]:=\{p, p+1, \cdots, q\}$ and we denote the resulting poset by $\Delta(\omega)$.

Remark 3.1 ([1]). The symmetric group $S_{n}$ is the Coxeter group of type $A_{n-1}$. Let $\Delta$ be the root system of type $A_{n-1}$ and $\left\{\alpha_{1}, \alpha_{2}, \cdots \alpha_{n-1}\right\}$ be a set of fundamental roots. The root system $\Delta$ is the disjoint union of the positive roots $\Delta^{+}$and the negative roots $\Delta^{-}$corresponding to the fundamental roots $\left\{\alpha_{1}, \alpha_{2}, \cdots \alpha_{n-1}\right\}$. Endow an order structure on $\Delta^{+}$such that $\alpha \leq \beta$ if and only if $\beta-\alpha \in \sum_{i} \mathbb{Z}_{\geq 0} \alpha_{i}$ and the resulting poset is called the root poset of type $A_{n-1}$. The symmetric group $S_{n}$ naturally acts on $\Delta\left(A_{n-1}\right)$. Let $N_{\omega}:=\left\{\alpha \in \Delta^{+} \mid \omega(\alpha) \in \Delta^{-}\right\}$. Then $N_{\omega}$ is a subposet of $\Delta^{+}$. Indeed $N_{\omega}$ is order isomorphic to $\Delta(\omega)$. Remark that $|\Delta(\omega)|=|\operatorname{Inv}(\omega)|$. 
It is natural to ask how the poset structures of $M_{\omega}$ and $\Delta(\omega)$ are related, because they are constructed by using the inversions of $\omega$.

Definition 3.1. We define a map $\phi_{\omega}: M_{\omega} \rightarrow \Delta(\omega)$ by

$$
\phi_{\omega}\left(m_{i, x}(\omega)\right)=\left(i, i_{x}\right)
$$

where $i<i_{1}<i_{2}<\cdots<i_{c_{i}(\omega)} \leq n$ with $\left(i, i_{1}\right),\left(i, i_{2}\right), \cdots,\left(i, i_{c_{i}(\omega)}\right) \in$ $\operatorname{Inv}(\omega)$.

There are $x-1$ zeros between $i$-th coordinate and $i_{x}$-th coordinate in $m_{i, x}(\omega)$, see Lemma 2.1. One can write

$$
\begin{array}{r}
\phi_{\omega}\left(\left(0, \cdots, 0, \stackrel{i-\mathrm{th}}{x}, x, x, \cdots, x, \stackrel{i_{1}-\text { th }}{0}, x-1, x-1, \cdots, x-1, \stackrel{i_{2}-\text { th }}{0}, \cdots, \stackrel{i_{x-1}-\text { th }}{0},\right.\right. \\
\left.\left.1, \cdots, 1, \stackrel{i_{x}-\text { th }}{0}, 0,0, \cdots, 0\right)\right)=\left(i, i_{x}\right) .
\end{array}
$$

Example 3.1. For $\omega=5371642$, we have

$$
\begin{aligned}
& \phi\left(m_{1,4}(5371642)\right)=(1,7), \quad \phi\left(m_{1,3}(5371642)\right)=(1,6), \\
& \phi\left(m_{1,2}(5371642)\right)=(1,4), \quad \phi\left(m_{1,1}(5371642)\right)=(1,2), \\
& \phi\left(m_{2,2}(5371642)\right)=(2,7), \quad \phi\left(m_{2,1}(5371642)\right)=(2,4), \\
& \phi\left(m_{3,4}(5371642)\right)=(3,7), \quad \phi\left(m_{3,3}(5371642)\right)=(3,6), \\
& \phi\left(m_{3,2}(5371642)\right)=(3,5), \quad \phi\left(m_{3,1}(5371642)\right)=(3,4), \\
& \phi\left(m_{5,2}(5371642)\right)=(5,7), \quad \phi\left(m_{5,1}(5371642)\right)=(5,6), \\
& \phi\left(m_{6,1}(5371642)\right)=(6,7) .
\end{aligned}
$$

Figure 9 shows the Hasse diagram of $\Delta(5371642)$.

The number of $M_{\omega}$ is that of inversions of $\omega$ and hence we have $\left|M_{\omega}\right|=$ $|\Delta(\omega)|=|\operatorname{Inv}(\omega)|$. Also $\phi_{\omega}$ is injective and therefore $\phi_{\omega}$ is a bijection.

We give the following useful statement, which is a straightforward consequence of Lemma 2.1 and we omit the proof.

Lemma 3.1. For $m_{i, x}(\omega) \in M_{\omega}$ with $1 \leq i<j \leq n$ and $x \in\left[c_{i}(\omega)\right]$, suppose that the $j$-th coordinate of $m_{i, x}(\omega)$ equals $y \geq 1$. Then the number of 0 between the $j$-th (resp. $i$-th) coordinate and the $i_{x}$-th (resp. $j$-th) coordinate 


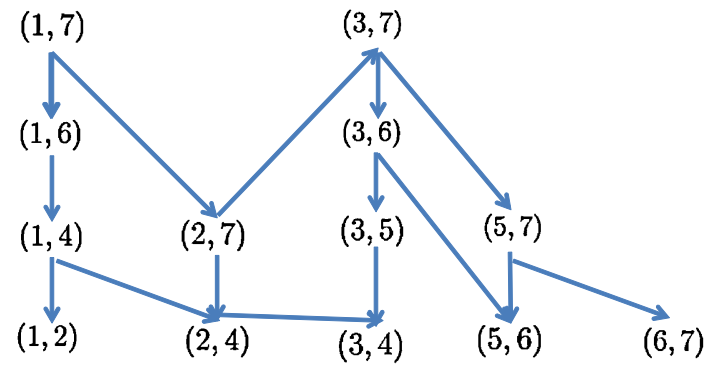

Figure 9: Hasse diagram of $\Delta(5371642)$.

The number of 0 is $y-1$

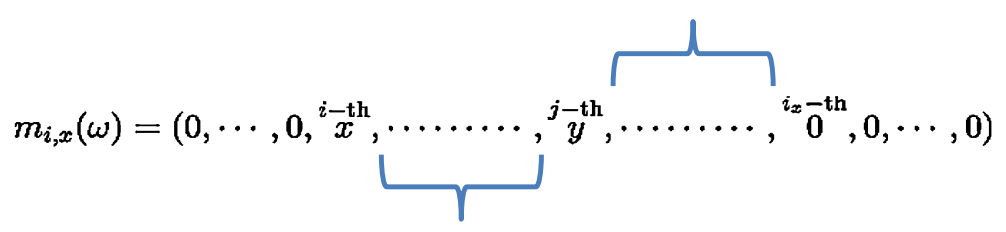

The number of 0 is $x-y$

Figure 10: A visualization of Lemma 3.1.

equals $y-1$ (resp. $x-y)$, where $\left(i, i_{1}\right),\left(i, i_{2}\right), \cdots,\left(i, i_{c_{i}(\omega)}\right)$ are the inversions of $\omega$ involving $i$ with $i_{1}<i_{2}<\cdots<i_{c_{i}(\omega)}$.

Figure 10 shows a visualization of Lemma 3.1.

Proposition 3.1. $\phi_{\omega}$ is an order-preserving bijection.

Proof. It suffices to show that $\phi_{\omega}$ is an order-preserving map. Suppose that $m_{i, x}(\omega)>m_{j, y}(\omega)$ for some $1 \leq i, j \leq n, x \in\left[c_{i}(\omega)\right]$ and $y \in\left[c_{j}(\omega)\right]$. Then $i \leq j$ by Lemma 1.1. We have to prove $i_{x} \geq j_{y}$.

If $i=j$, then we have $x>y$ by Lemma 1.2 and we see $i_{x}>j_{y}$. Hence $\phi_{\omega}\left(m_{i, x}(\omega)\right)=\left(i, i_{x}\right) \succ \phi_{\omega}\left(m_{i, y}(\omega)\right)=\left(i, i_{y}\right)$.

Now we suppose $i<j$.

Because $m_{i, x}(\omega)>m_{j, y}(\omega)$, the $j$-th coordinate of $m_{i, x}(\omega)$ is greater than or equal to $y$ which is not 0 . On the other hand every $k$-th coordinate with $i_{x} \leq k$ equals 0 . So we find $j<i_{x}$.

We can write

$$
m_{i, x}(\omega)=\left(0, \cdots, 0, \stackrel{i-\text { th }}{x}, \cdots, \stackrel{j-\text { th }}{z}, \cdots, \stackrel{i_{x}-\text { th }}{0}, \cdots 0\right)
$$


The number of 0 is $z-1$

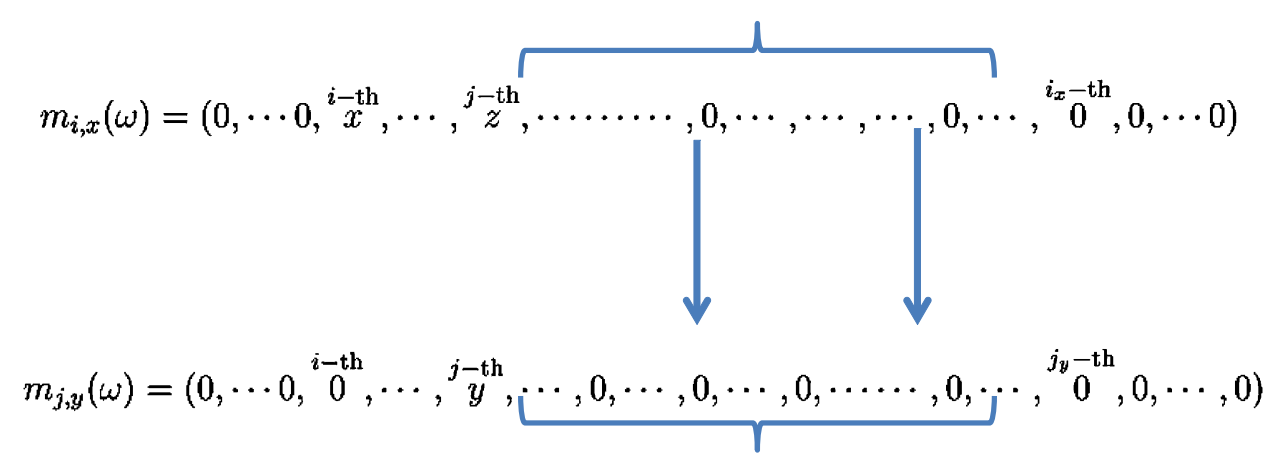

The number of 0 is $y-1$

Figure 11: Illustration of the proof of Proposition 3.1.

and

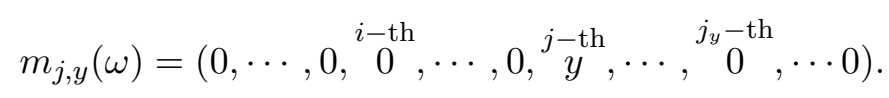

for some $x \geq z \geq y \geq 1$. Also we have $\omega(i)<\omega(j)$ by Lemma 1.3.

For $j<k \leq i_{x}$, if the $k$-th coordinate of $m_{i, x}(\omega)$ is 0 , then $\omega(j)>\omega(i)>$ $\omega(k)$ and hence the $k$-th coordinate of $m_{j, y}(\omega)$ equals 0 .

If $j_{y}>i_{x}$, then the number of 0 between $j$-th coordinate and $j_{y}$-th coordinate in $m_{j, y}(\omega)$, which equals $y-1$ by Lemma 3.1, is greater than that of 0 between $j$-th coordinate and $i_{x}$-th coordinate in $m_{i, x}(\omega)$, which equals $z-1$ by Lemma 3.1, because the $i_{x}$-th coordinate of $m_{j, y}(\omega)$ is 0 , see Figure 11. This contradicts the assumption that $m_{i, x}(\omega)>m_{j, y}(\omega)$. Hence we have $j_{y} \leq i_{x}$ and $\left(i, i_{x}\right) \succ\left(j, j_{y}\right)$ in $\Delta(\omega)$. Now we conclude that $\phi_{\omega}$ preserves the order structure.

It is natural to ask when $\phi_{\omega}$ becomes a poset isomorphism.

Proposition 3.2. If $\omega \in S_{n}$ is a 321-avoiding permutation, then $\phi_{\omega}$ is a poset isomorphism.

Proof. Because $\phi_{\omega}$ is an order-preserving bijection, it suffices to show that $\phi_{\omega}^{-1}$ is an order-preserving map. Suppose that $\phi_{\omega}\left(m_{i, x}(\omega)\right) \succ \phi_{\omega}\left(m_{j, y}(\omega)\right)$ in $\Delta(\omega)$, where $m_{i, x}(\omega), m_{j, y}(\omega) \in M_{\omega}$ with $1 \leq i, j \leq n, x \in\left[c_{i}(\omega)\right]$ and $y \in\left[c_{j}(\omega)\right]$. It suffices to prove $m_{i, x}(\omega)>m_{j, y}(\omega)$ in $M_{\omega}$. Put $\phi_{\omega}\left(m_{i, x}(\omega)\right)=$ $\left(i . i_{x}\right)$ and $\phi_{\omega}\left(m_{j, y}(\omega)\right)=\left(j, j_{y}\right)$ with $i \leq j<j_{y} \leq i_{x}$. 
$j_{y} \leq i_{x}$

$m_{i, x}(\omega)=\left(0, \cdots, 0, \stackrel{i-\text { th }}{x}, \cdots, \stackrel{j-\text { th }}{z}, \cdots \cdots, 0, \cdots, 0, \cdots, 0, \cdots, 0, \cdots, \stackrel{i_{x}-\text { th }}{0}, 0, \cdots 0\right)$

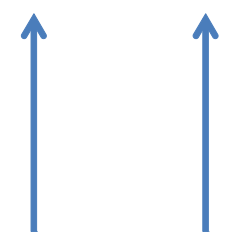

$m_{j, y}(\omega)=\left(0, \cdots, 0, \stackrel{i-\text { th }}{0}, \cdots,{ }^{j-\text { th }} y, \cdots \cdots, 0, \cdots \cdots, 0, \cdots \cdots, \stackrel{j_{y} \text {-th }}{0}, 0, \cdots \cdots, 0\right)$

Figure 12: Illustration of Claim 2 and Claim 3 of Proposition 3.2.

Case 1: $i=j$.

We see $i=j<j_{y}<i_{x}$ and $j_{y}=i_{y}$. Then $i_{y}<i_{x}$ and we have $y<x$. By Lemma 1.2, we obtain $m_{i, x}(\omega)>m_{i, y}(\omega)$ in $M_{\omega}$.

Case 2: $i<j$.

We have $i<j<j_{y} \leq i_{x}$. Write

$$
m_{i, x}(\omega)=\left(0, \cdots, 0, \stackrel{i-\text { th }}{x}, \cdots, \stackrel{j-\text { th }}{z}, \cdots, \stackrel{i_{x}-\text { th }}{0}, 0, \cdots, 0\right)
$$

and

$$
m_{j, y}(\omega)=\left(0, \cdots, 0, \stackrel{i-\text { th }}{0}, \cdots, \stackrel{j-\text { th }}{y}, \cdots, \stackrel{j_{y}-\text { th }}{0}, 0, \cdots, 0\right) .
$$

for some $z \geq 0$.

\section{Claim 1}

$$
\omega(i)<\omega(j) .
$$

Proof of Claim 1. If $\omega(i)>\omega(j)$, then $\operatorname{st}\left(\omega(i) \omega(j) \omega\left(j_{y}\right)\right)=321$ and this contradicts the assumption.

\section{Claim 2}

For $j<k \leq j_{y}$, if the $k$-th coordinate of $m_{j, y}(\omega)$ equals 0 , then the $k$-th coordinate of $m_{i, x}(\omega)$ is 0 , see Figure 12 .

Proof of Claim 2. Suppose that $j_{y}=i_{x}$. Then the $j_{y}$-th coordinate of $m_{j, y}(\omega)$ and that of $m_{i, x}(\omega)$ equal 0 . If $j<k<j_{y}$ and the $k$-th coordinate of $m_{j, y}(\omega)$ equals 0 , then $\omega(j)>\omega(k)$ by Lemma 2.1. Because $\omega(j)>\omega(k)$ 
and $\operatorname{st}\left(\omega(j) \omega(k) \omega\left(i_{x}\right)\right) \neq 321$, we have $\omega(k)<\omega\left(i_{x}\right)<\omega(i)$. Therefore the $k$-th coordinate of $m_{i, x}(\omega)$ equals 0 .

Suppose that $j_{y}<i_{x}, j<k \leq j_{y}$ and the $k$-th coordinate of $m_{j, y}(\omega)$ equals 0. Because $\omega(j)>\omega(k)$ and $s t\left(\omega(j) \omega(k) \omega\left(i_{x}\right)\right) \neq 321$, we have $\omega(k)<$ $\omega\left(i_{x}\right)<\omega(i)$. Therefore the $k$-th coordinate of $m_{i, x}(\omega)$ equals 0 .

\section{Claim 3}

For $j \leq k \leq j_{y}$, if the $k$-th coordinate of $m_{j, y}(\omega)$ is not 0 , then the $k$-th coordinate of $m_{i, x}(\omega)$ is greater than or equal to that of $m_{j, y}(\omega)$.

Proof of Claim 3. By assumption, we have $\omega(j)<\omega(k)$ by Lemma 2.1 and the $k$-th coordinate of $m_{j, y}(\omega)$ equals the number of 0 between $k$-th coordinate and $j_{y}$-th coordinate +1 by Lemma 3.1. Also we see $\omega(i)<\omega(k)$ by Claim 1 and the $k$-th coordinate of $m_{i, x}(\omega)$ is also the number of 0 between $k$-th coordinate and $i_{x}$-th coordinate +1 by Lemma 3.1. Because $j_{y} \leq i_{x}$ and Claim 2, the number of 0 between $k$-th coordinate and $i_{x}$-th coordinate of $m_{i, x}(\omega)$ is greater than or equal to that of 0 between $k$-th coordinate and $j_{y}$-th coordinate of $m_{j, y}(\omega)$.

If $k<j$ or $j_{y} \leq k$, then the $k$-th coordinate of $m_{j, y}(\omega)$, which is 0 , is less than or equal to that of $m_{i, x}(\omega)$.

Also for $j \leq k \leq j_{y}$, from the above argument, the $k$-th coordinate of $m_{j, y}(\omega)$ is less than or equal to that of $m_{i, x}(\omega)$ by Claim 3 . Hence $m_{i, x}(\omega)>$ $m_{j, y}(\omega)$ in $M_{\omega}$. Now we conclude that if $\omega$ is a 321-avoiding permutation, then $\phi_{\omega}$ is a poset isomorphism.

Now we obtain the following result.

Theorem 3.1. For $\omega \in S_{n}, \phi_{\omega}$ is a poset isomorphism if and only if $\omega$ is a 321-avoiding permutation.

Proof. It suffices to prove that if $\omega$ has a 321-pattern, then $\phi_{\omega}$ is not a poset isomorphism. By assumption, there exists $1 \leq i<j<k \leq n$ such that $\omega(i)>\omega(j)>\omega(k)$. Without loss of generality, we can assume that $\omega(j)<\omega(p)$ for all $j<p<k$.

Put $m_{i, x}(\omega):=\phi_{\omega}^{-1}((i, k))$. We remark that $(i, j),(i, k) \in \Delta(\omega)$ and the $j$-th coordinate of $m_{i, x}(\omega)$ is 0 . We write

$$
m_{i, x}(\omega)=(0, \cdots, 0, \stackrel{i-\text { th }}{x}, \cdots, \stackrel{j-\text { th }}{0}, \cdots, \stackrel{k-\text { th }}{0}, 0, \cdots, 0)
$$

and

$$
m_{j, 1}(\omega)=(0, \cdots, 0, \stackrel{i-\text { th }}{0}, \cdots, 0, \stackrel{j-\text { th }}{1}, 1, \cdots, 1, \stackrel{k-\text { th }}{0}, 0, \cdots, 0) .
$$


For $j \leq p<k$, the $p$-th coordinate of $m_{j, 1}(\omega)$ equals 1 because $\omega(j)<$ $\omega(p)$ and by Lemma 2.1 .

Now we have $\phi_{\omega}\left(m_{i, x}(\omega)\right)=(i, k) \succ(j, k)=\phi_{\omega}\left(m_{j, 1}(\omega)\right)$ in $\Delta(\omega)$. On the other hand, $m_{i, x}(\omega)$ and $m_{j, 1}(\omega)$ are incomparable by comparing their $i$ th coordinates and $j$-th coordinates. We conclude that if $\omega$ has a 321-pattern, then $\phi_{\omega}$ is not poset isomorphism.

\section{Acknowledgment}

The author wishes to thank Hugh Denoncourt and Kento Nakada for their valuable advice. He is also grateful for two anonymous referees for their improvement of the paper.

\section{References}

[1] A. Björner, F. Brenti, Combinatorics of Coxeter Groups, SpringerVerlag, New York, 2005. MR2133266

[2] M. Bóna, Combinatorics of Permutations, CRC Press/Chapman Hall, Boca Raton, FL, 2004. MR2078910

[3] H. Denoncourt, A refinement of weak order intervals into distributive lattices, Annals of Combinatorics, 17 (2013), 655-670. MR3129777

[4] D. Knuth, The Art of Computer Programming, I: Fundamental Algorithms, Addison-Wesley, Publishing Co, Reading, Mass-London-Don Mills, Ont, 1969. MR0378456

[5] D. Knuth, The Art of Computer Programming, III: Sorting and Searching, Addison-Wesley, Reading, MA, 1973. MR0378456

[6] D. H. Lehmer, Teaching combinatorial tricks to a computer, in Proc. Sympos. Appl. Math., 10 (1960), Amer. Math. Soc., 179-193. MR0113289

[7] R. P. Stanley, Enumerative Combinatorics, vol. 1, Chapter 3, Cambridge University Press, Cambridge, 1999. MR1442260

Masaya TOMIE

MORIOKA UNIVERSITY

TAKIZAWA-SHI, IWATE 020-0183

JAPAN

E-mail address: tomie@morioka-u.ac.jp

RECEIVED 31 JANUARY 2014 